6-9-2011

\title{
A Direct Sampling Particle Filter from Approximate Conditional Density Function Supported on Constrained State Space
}

Sridhar Ungarala

Cleveland State University

Follow this and additional works at: https://engagedscholarship.csuohio.edu/encbe_facpub

Part of the Process Control and Systems Commons

How does access to this work benefit you? Let us know!

\section{Publisher's Statement}

NOTICE: this is the author's version of a work that was accepted for publication in Computers \& Chemical Engineering. Changes resulting from the publishing process, such as peer review, editing, corrections, structural formatting, and other quality control mechanisms may not be reflected in this document. Changes may have been made to this work since it was submitted for publication. A definitive version was subsequently published in Computers \& Chemical Engineering, 35, 6, (June 9, 2011) DOI 10.1016/j.compchemeng.2010.07.022

\section{Original Citation}

Ungarala, S. (2011). A direct sampling particle filter from approximate conditional density function supported on constrained state space. Computers and Chemical Engineering, 35(6), 1110-1118. doi:10.1016/j.compchemeng.2010.07.022

\section{Repository Citation}

Ungarala, Sridhar, "A Direct Sampling Particle Filter from Approximate Conditional Density Function Supported on Constrained State Space" (2011). Chemical \& Biomedical Engineering Faculty Publications. 73.

https://engagedscholarship.csuohio.edu/encbe_facpub/73

This Article is brought to you for free and open access by the Chemical \& Biomedical Engineering Department at EngagedScholarship@CSU. It has been accepted for inclusion in Chemical \& Biomedical Engineering Faculty Publications by an authorized administrator of EngagedScholarship@CSU. For more information, please contact library.es@csuohio.edu. 


\title{
A direct sampling particle filter from approximate conditional density function supported on constrained state space
}

\author{
Sridhar Ungarala* \\ Department of Chemical and Biomedical Engineering, 2121 Euclid Avenue, Cleveland State University, Cleveland, OH 44115, USA
}

\section{Introduction}

This paper is concerned with state estimation of nonlinear dynamic systems, where the state vector is subject to constraints. These constraints may occur in the form of multivariate algebraic equality and inequality relationships, which may be linear or nonlinear. Constrained state estimation is commonly dealt with in an optimization framework by minimizing a cost function over constrained state space. The moving horizon estimation (MHE) is an example of this strategy (Rao, \& Rawlings, 2002; Robertson, Lee, \& Rawlings, 1996). There are two open issues in practical implementation of MHE, viz., (1) the computation of arrival cost using approximate, unconstrained mean and covariance at the beginning of each data window and (2) the appropriate size of the moving window to balance performance and computational load. As a means to address these issues, Rawlings and Bakshi (2006) indicated potential synergies between MHE and recent advances in sequential Monte Carlo methods known as particle filters (PF) for state estimation.

Particle filters have gained wide ranging audience due to their ability to circumvent functional approximations of nonlinear models and Gaussian approximations of probability density function (pdf) (Arulampalam, Maskell, Gordon, \& Clapp, 2002; Chen, Bakshi, Goel, \& Ungarala, 2004; Gordon, Salmond, \& Smith, 1993). The filter works with a set of weighted samples representing the state conditional pdf, while recursively updating them using importance sampling and resampling techniques. The use of MHE for correcting the behavior of particle filter initialized by poor quality information about the initial condition of the system is suggested by several authors (Botchu, 2006; Lang, Goel, \& Bakshi, 2006; Lang, Zhang, Goel, \& Bakshi, 2005; Rajamani, \& Rawlings, 2007). Likewise, the use of particle filter to accurately propagate arrival cost parameters in MHE is also recommended as a means to keep the horizon length small (Ungarala, 2009). In related work the unscented Kalman filter (UKF) is used for arrival cost (Qu \& Hahn, 2009).

There is research reported on constrained state estimation using the underlying Monte Carlo approach. Recently Lang, Chen, Bakshi, Goel, and Ungarala (2007) presented a modification to PF by introducing additional acceptance/rejection steps into the generic PF algorithm in order to discard samples that violated the constraints. This method is limited to simple upper and lower bounds on the variables. Unconstrained sampling followed by verification against applicable constraints can be taxing on resources when compared to directly sampling from the constrained state space. One such technique is the cell filter where a Markov chain is constructed by sampling the dynamics over constraints (Ungarala, Li, \& Chen, 2008), however, this approach is limited to low dimensional systems due to exponentially increasing memory requirements of the state transition operator with the state dimension.

Other sampling based methods similar to the particle filter have been used for imposing constraints by projection techniques. See Simon (2010) for a survey of projection based approaches to constrained estimation in the Kalman filter framework. The constraint violating sigma points and mean vectors are projected to constraint boundary at the prediction and update stages of UKF (Kandepu, Foss, \& Imsland, 2008). Julier and LaViola (2007) pre- 
sented a detailed discussion on the need for projection operations both on samples and moments when nonlinear constraints are encountered.

In related work, constrained optimization is used to recursively update the samples of the conditional density. The unscented recursive nonlinear dynamic data reconciliation (URNDDR) method used a weighted least squares objective function to update the UKF sigma points (Narasimhan \& Rengaswamy, 2009; Vachhani, Narasimhan, \& Rengaswamy, 2006). Similarly, samples of the a priori density in the ensemble Kalman filter (EnKF) (Prakash, Patwardhan, \& Shah, 2010) and the particle filter (Prakash, Patwardhan, \& Shah, 2008; Shao, Huang, \& Lee, 2010) are updated by the least squares formulation applied independently to each sample. This approach is also termed as nonlinear programming filters (Kolas, Foss, \& Schei, 2009) and interval constrained filters (Teixeira, Torres, Aguirre, \& Bernstein, 2010). These methods are based on a common assumption that the a priori density is a multivariate Gaussian pdf.

The assumption of Gaussianity of the prior pdf is not needed in the generic particle filter. If such an assumption is inserted in the PF, it is submitted in this paper that samples can be drawn directly from the resulting approximate conditional density. Furthermore, the sampling process can be restricted to constrained state space. In this manner, importance sampling and sampling by acceptance/rejection method is avoided. This approach can be computationally less expensive when compared to optimization of samples under constraints.

The proposed approach is called direct sampling particle filter (DSPF) from an approximate conditional density supported on constrained state space. For linear constraints, the mean of the constrained samples, as the state estimate, automatically respects the constraints due to the superposition principle. In linearly constrained nonlinear systems the DSPF can be used to provide constrained mean and covariance for the arrival cost computation in MHE.

When samples are drawn from nonlinear constraints, there is no guarantee that the sample mean will be constrained. It is proposed to use the constrained mode as the state estimate by solving the maximum a posteriori problem. Alternatively, the unconstrained mean is projected on constraints to yield an estimate with a larger variance.

In the following, Bayesian state estimation problem and the particle filter are summarized. Direct sampling from approximate conditional density is discussed including linear and nonlinear constraints and the DSPF algorithm. Three simulation examples are included at the end involving linear and nonlinear equality constraints, linear inequality constraints as well as linear and nonlinear measurements. A fourth example demonstrates the poor performance of DSPF in highly nonlinear systems, where a Gaussian approximation of multimodal prior is shown to be detrimental to performance.

\section{State estimation}

Let a general form of discrete-time nonlinear dynamic system driven by additive zero mean Gaussian noise be chosen as

$x_{k}=f\left(x_{k-1}\right)+w_{k-1}$,

where $f: \mathbb{R}^{n} \rightarrow \mathbb{R}^{n}$ is a nonlinear vector function of the state $x_{k}$ and $w_{k} \sim \mathcal{N}(0, Q)$. A corresponding model for the dynamics of the state probability density function $p\left(x_{k}\right)$ is the transition probability density $p\left(x_{k} \mid x_{k-1}\right)$, which is derived from the state transition equation and system noise pdf as

$$
p\left(x_{k} \mid x_{k-1}\right)=\frac{1}{(2 \pi)^{n / 2}|Q|^{1 / 2}} e^{-1 / 2\left\|x_{k}-f\left(x_{k-1}\right)\right\|_{Q^{-1}}^{2}} .
$$

The temporal evolution of the state pdf is given by the Chapman-Kolmogorov equation

$p\left(x_{k}\right)=\int p\left(x_{k} \mid x_{k-1}\right) p\left(x_{k-1}\right) \mathrm{d} x_{k-1}$,

initialized with $p\left(x_{0}\right)$, which is typically assumed as a Gaussian $\mathcal{N}\left(\hat{x}_{0}, \hat{P}_{0}\right)$.

Noisy measurements of the process $y_{k}$ are related to the state vector as

$y_{k}=h\left(x_{k}\right)+v_{k}$,

where $h: \mathbb{R}^{n} \rightarrow \mathbb{R}^{p}$ is a nonlinear vector function and $v_{k} \sim \mathcal{N}(0, R)$. In probabilistic terms, the relationship between the state space and a given measurement is expressed by the likelihood function $p\left(y_{k} \mid x_{k}\right)$, which is derived from the measurement equation and measurement noise pdf as

$p\left(y_{k} \mid x_{k}\right)=\frac{1}{(2 \pi)^{p / 2}|R|^{1 / 2}} e^{-1 / 2\left\|y_{k}-h\left(x_{k}\right)\right\|_{R^{-1}}^{2}}$.

Given the history of measurements $Y_{1: k}$, it is desired to estimate the current state of the system. The solution is to construct the conditional probability density function $p\left(x_{k} \mid Y_{1: k}\right)$. The state estimate is then drawn as a conditional inference from this pdf. The conditionally expected value of a real valued vector function $\phi\left(x_{k}\right)$ is computed by

$E\left[\phi\left(x_{k}\right) \mid Y_{1: k}\right]=\int \phi\left(x_{k}\right) p\left(x_{k} \mid Y_{1: k}\right) \mathrm{d} x_{k}$.

The mean and covariance are typically used for state estimation. This computation requires a knowledge of the conditional density function at each time instance.

A combination of Chapman-Kolmogorov equation and Bayes theorem provides a means to recursively compute the conditional density function as follows:

$p\left(x_{k} \mid Y_{1: k}\right)=\frac{p\left(y_{k} \mid x_{k}\right) \int p\left(x_{k} \mid x_{k-1}\right) p\left(x_{k-1} \mid Y_{1: k-1}\right) \mathrm{d} x_{k-1}}{\int p\left(y_{k} \mid \xi_{k}\right) p\left(\xi_{k} \mid Y_{1: k-1}\right) \mathrm{d} \xi_{k}}$,

where the integral term in the numerator is computing the a priori density $p\left(x_{k} \mid Y_{1: k-1}\right)$ and the denominator integral is a normalizing constant independent of $x_{k}$. Generally it is not possible to find analytical forms of the conditional density without using simplifying assumptions.

\section{Particle filter}

The particle filter is a broad class of methods that implement in spirit Bayesian recursion of conditional density in terms of weighted samples of the density. It is rooted in Monte Carlo approximation of expectation operations using sample averages. Suppose that the set $\left\{x^{i}\right\}_{i=1}^{N}$ contains random samples of state vector distributed according to the pdf $p(x)$. An expectation operation such as in Eq. (6) is approximated by

$E[\phi(x)] \approx \frac{1}{N} \sum_{i=1}^{N} \phi\left(x^{i}\right)$

Generally, it may not be possible to draw samples directly from the desired density $p(x)$. Another function with a similar shape that includes the support of the desired density may be chosen for sampling. This is known as importance or proposal density function 
$q(x)$. A weighted mean of samples drawn from $q(x)$ then approximates the desired expectation as

$E[\phi(x)] \approx u_{i=1}^{N} u^{i} \phi\left(x^{i}\right)$,

where the importance weights $u^{i}$ are defined as

$$
\begin{aligned}
& u^{i}=\frac{p\left(x^{i}\right) / q\left(x^{i}\right)}{N} . \\
& p\left(x^{i}\right) / q\left(x^{i}\right) \\
& i=1
\end{aligned}
$$

Sequential importance sampling is generally the basis for most particle filters where samples are propagated forward, importance weights are updated by measurements and Monte Carlo averages provide state estimates. Because of its central role in a particle filter, the choice of importance density is critical. One of the common choices is the transition density $p\left(x_{k} \mid x_{k-1}\right)$, also referred to as prior importance density because when conditioned on available measurements it is also $p\left(x_{k} \mid Y_{1: k-1}\right)$, the a priori density (Gordon et al., 1993).

Generating samples of the prior importance density is straightforward. Let the set $\left\{\hat{x}_{k-1}^{i}\right\}_{i=1}^{N}$ contain random samples of the state vector distributed according to the conditional pdf $p\left(x_{k-1} \mid Y_{1: k-1}\right)$. A set of random vectors $\left\{w_{k-1}^{i}\right\}_{i=1}^{N}$ is sampled from $\mathcal{N}(0, Q)$, and the system model is used to make $N$ state transitions in parallel

$\tilde{x}_{k}^{i}=f\left(\hat{x}_{k-1}^{i}\right)+w_{k-1}^{i}, \quad i=1, \ldots, N$.

The transformed samples $\left\{\tilde{x}_{k}^{i}\right\}_{i=1}^{N}$ will be distributed according to $p\left(x_{k} \mid Y_{1: k-1}\right)$, chosen as the importance density.

The likelihood of the predicted samples is represented by importance weights $\left\{u_{k}^{i}\right\}_{i=1}^{N}$. Given the measurement $y_{k}$, the weights are computed by

$$
\begin{aligned}
& u_{k}^{i}=\frac{p\left(y_{k} \mid \tilde{x}_{k}^{i}\right)}{N}, \\
& p\left(y_{k} \mid \tilde{x}_{k}^{i}\right) \\
& i=1
\end{aligned}
$$

which requires the likelihood function for point-wise evaluation as in Eq. (5). The importance weights represent relative probabilities of the samples and they constitute a discrete distribution with probability mass $u_{k}^{i}$ supported on $\tilde{x}_{k}^{i}$. A resampled set $\left\{\hat{x}_{k}^{i}\right\}_{i=1}^{N}$ is formed by drawing $N$ samples from the discrete distribution such that $\operatorname{Pr}\left[\hat{x}_{k}^{j}=\tilde{x}_{k}^{i}\right]=u_{k}^{i}$. After resampling, the importance weights are reset to $u^{i}=1 / N, \forall i$. The sample statistics provide approximations to the desired conditional moments as state estimates.

\section{Approximate conditional density}

Samples of the set $\left\{\tilde{x}_{k}^{i}\right\}_{i=1}^{N}$ predicted by the system model in Eq. (11) are distributed according to the $a$ priori density $p\left(x_{k} \mid Y_{1: k-1}\right)$. It is generally a time-varying non-Gaussian density that is translated and distorted each time. Suppose this a priori pdf is approximated as a fixed-shape multivariate Gaussian pdf whose time-varying statistics are

$\tilde{x}_{k}=\frac{1}{N} \tilde{x}_{i=1}^{N}$,

$\tilde{P}_{k}=\frac{1}{N-1}{ }_{i=1}^{N}\left(\tilde{x}_{k}^{i}-\tilde{x}_{k}\right)\left(\tilde{x}_{k}^{i}-\tilde{x}_{k}\right)^{\mathrm{T}}$.
Consequently, the closed-form approximation of the a priori probability density is

$p^{*}\left(x_{k} \mid Y_{1: k-1}\right)=\frac{1}{(2 \pi)^{n / 2}\left|\tilde{P}_{k}\right|^{1 / 2}} e^{-1 / 2 x_{k}-\tilde{x}_{k}} \tilde{P}_{k}^{2}$.

Now a closed-form approximation of the conditional density $p\left(x_{k} \mid Y_{1: k}\right)$ is determined by Bayes theorem. The approximate conditional density denoted as $p^{*}\left(x_{k} \mid Y_{1: k}\right)$ is a normalized product of $p^{*}\left(x_{k} \mid Y_{1: k-1}\right)$ above and $p\left(y_{k} \mid x_{k}\right)$ in Eq. (5)

$\left.p^{*}\left(x_{k} \mid Y_{1: k}\right)=\frac{1}{c} e^{-1 / 2\left(x_{k}-\tilde{x}_{k}\right)} \underset{\tilde{P}_{k}^{-1}}{2}+\| y_{k}-h\left(x_{k}\right)_{R^{-1}}^{2}\right)$,

where $c$ in the denominator is a normalizing constant given as the integral

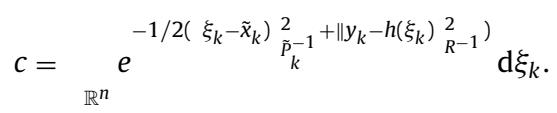

Although the above integration for the normalizing constant has the domain $\mathbb{R}^{n}$ in theory, for practical implementation it can be limited to $\mathbb{R}_{\Sigma}^{n}$ that includes upper and lower bounds on each dimension of the state space. With a knowledge of the a priori mean and covariance, the bounds on $\mathbb{R}_{\Sigma}^{n}$ are chosen using the 3-sigma rule for the support of a Gaussian pdf that states that $99.7 \%$ of its samples lie within three standard deviations of the mean. The 4-sigma based bounds will cover $99.99 \%$ of samples, which is useful for a wider exploration of the state space when prior information is deemed of poor quality. The bounds are meaningful because the support of the conditional density is a subset of the support of the a priori density.

Suppose the samples $\left\{x_{k}^{i}\right\}_{i=1}^{N}$ are uniformly sampled over $\mathbb{R}_{\Sigma}^{n}$, called as candidate samples. The approximate conditional density evaluated at each candidate sample as $p^{*}\left(x_{k}^{i} \mid Y_{1: k}\right)$ and subsequently normalized, yields a discrete distribution. Thereafter, a discrete cumulative distribution function is computed for the candidate samples. It is then possible to generate samples $\left\{\hat{x}_{k}^{i}\right\}_{i=1}^{N}$ distributed according to $p^{*}\left(x_{k} \mid Y_{1: k}\right)$, from the uniformly assembled candidate samples. This approach is termed as direct sampling particle filter (DSPF) from an approximate conditional density. The sample statistics are used for state estimation as follows:

$\hat{x}_{k}=\frac{1}{N} \hat{x}_{i=1}^{N}$,

$\hat{P}_{k}=\frac{1}{N-1}{ }_{i=1}^{N}\left(\hat{x}_{k}^{i}-\hat{x}_{k}\right)\left(\hat{x}_{k}^{i}-\hat{x}_{k}\right)^{\mathrm{T}}$,

and the samples are propagated forward for the next measurement.

\subsection{Linear constraints}

Consider linear equality constraints such that

$\mathbb{X}=\{x: A x=b\}$,

or linear inequality constraints in the form of

$\mathbb{X}=\{x: A x \geq b\}$.

The a priori samples $\left\{\tilde{x}_{k}^{i}\right\}_{i=1}^{N}$ from Eq. (11) will not be typically constrained because the system model is unaware of the constraints and also because of the stochastic excitation. The constraint violating samples can be projected on the constraint border such as a hyper-plane, using a projection operator $\mathbf{p}$. Then samples $\tilde{x}_{k}^{i}$ violating the constraint are replaced by projections $\tilde{x}_{k}^{i, p}$

$\tilde{x}_{k}^{i, p}=\mathbf{p}\left(\tilde{x}_{k}^{i}\right)$. 
A straightforward projection method is one that minimizes the Euclidean distance of a sample from the constraint surface. The projection operator is valid for the unconstrained a priori mean because a linear constraint subsumes a linear combination of the samples such as their mean. Since the $a$ priori density is approximated as a Gaussian, it is sufficient to obtain the projected mean $\tilde{x}_{k}^{p}$ and the corresponding covariance matrix $\tilde{P}_{k}^{p}$. Details of a least squares projection operator are shown in Appendix A. See Simon (2010) for a survey of projection based methods.

In the presence of constraints $\mathbb{X}$, an approximate conditional density $p_{\mathbb{X}}^{*}\left(x_{k} \mid Y_{1: k}\right)$ is supported only on the constraints and has a value of zero everywhere else in the state space. As a result, the normalizing constant of $p_{\mathbb{X}}^{*}\left(x_{k} \mid Y_{1: k}\right)$ is determined by integrating over the domain $\mathbb{X}$, i.e. candidate samples are drawn uniformly from $\mathbb{X}$. The samples $\left\{\hat{x}_{k}^{i}\right\}_{i=1}^{N}$ drawn according to the discrete cumulative distribution function evaluated on the candidate samples will belong to $\mathbb{X}$. Consequently, the mean and covariance of the samples $\left\{\hat{x}_{k}^{i}\right\}_{i=1}^{N}$ will reflect the constraints. In practice, the candidate samples are drawn from the state space $\mathbb{R}_{\Sigma}^{n} \cap \mathbb{X}$, because the support of the assumedly Gaussian prior is also practically bound by 3-sigma limits.

The proposed approach is referred to as direct sampling particle filter from an approximate conditional density supported over constrained state space. When using the MHE approach to this constrained problem, the constrained mode is used as the state estimate but the mean and covariance are typically left unconstrained by the suboptimal nonlinear filters used for arrival cost such as EKF, UKF and PF (Qu \& Hahn, 2009; Robertson \& Lee, 1995; Ungarala, 2009). The DSPF can remedy this situation by propagating a constrained arrival cost for MHE.

\subsection{Nonlinear constraints}

General nonlinear equality constraints of the form

$\mathbb{X}=\{x: g(x)=0\}$

are considered and may be extended to inequality constraints as well. The use of nonlinear constraints with the a priori samples is complicated. Once again the violating samples may be projected on the constraint surface, however the mean is not guaranteed to be a member of $\mathbb{X}$ because the principle of superposition is not valid in this case. Although the mean violates constraints, projection of samples reduces the covariance due to additional information from the constraints. Therefore, the statistics of the constrained sample set are preferable to those of the unconstrained predicted sample set. The mean can also be subsequently projected on the constraint surface, however this step increases the covariance because the projected mean does not satisfy the minimum variance criterion under the stated assumptions (Julier \& LaViola, 2007).

The same problem arises for the statistics of samples from the constrained approximate conditional density. It is meaningful to consider the approximate conditional density defined only over support characterized by nonlinear constraints $\mathbb{X}$. However, the projected mean estimate is not an approximation of the minimum variance estimate under the stated assumptions.

An alternative approach for constrained state estimation is to use the mode of the constrained approximate conditional density $p_{\mathbb{X}}^{*}\left(x_{k} \mid Y_{1: k}\right)$ as the state estimate in a maximum a posteriori (MAP) sense. The mode belongs to $\mathbb{X}$ and it makes an acceptable state estimate. In this manner, the projection operation on the mean is avoided in the measurement update. The mode $\bar{x}_{k}$ is located by the following optimization problem:

$$
\begin{aligned}
\bar{x}_{k}= & \operatorname{argmax}_{x_{k}} p_{\mathbb{X}}^{*}\left(x_{k} \mid Y_{1: k}\right), \\
& \text { s.t. } x_{k} \in \mathbb{R}_{\Sigma}^{n} \cap \mathbb{X} .
\end{aligned}
$$

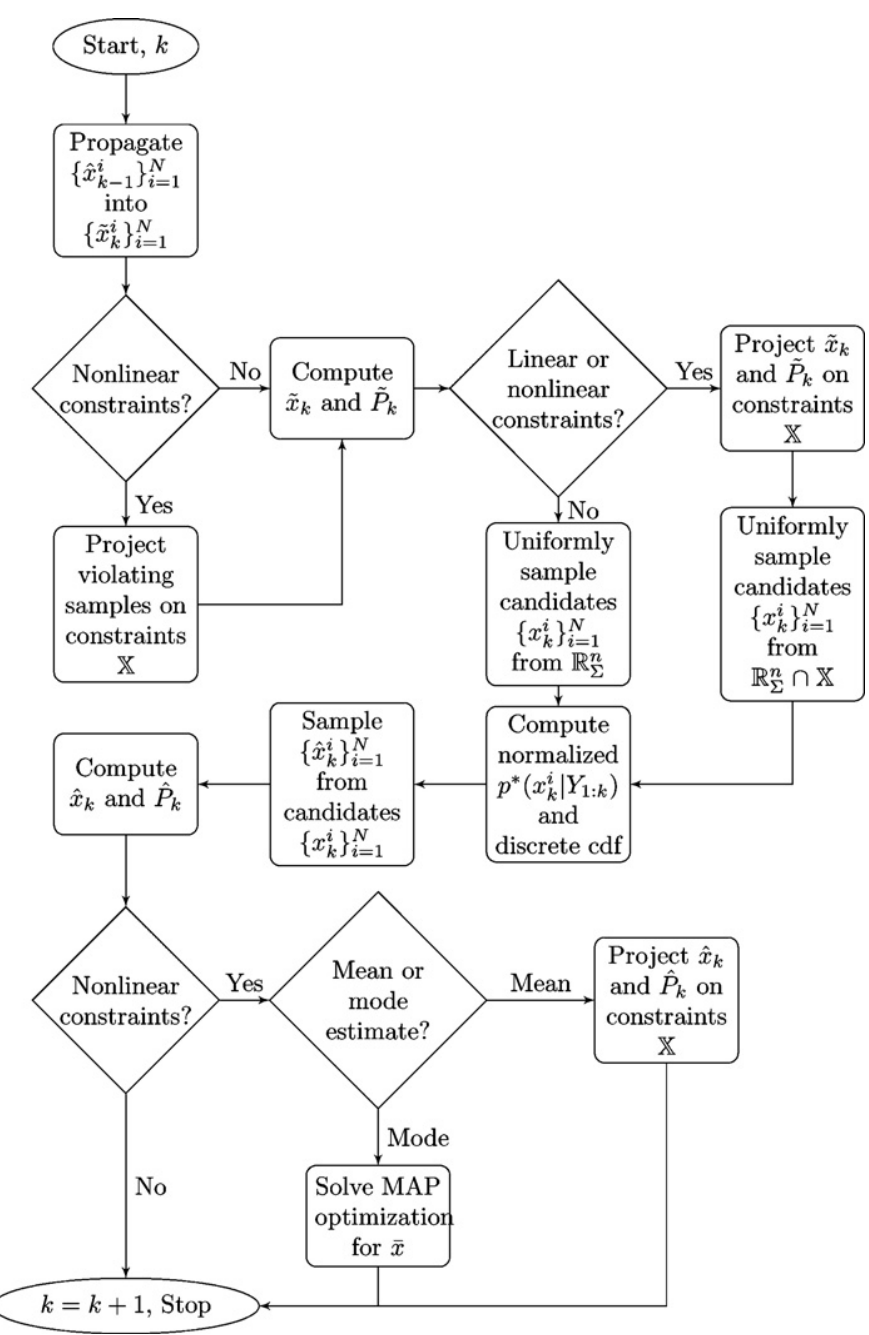

Fig. 1. Flowchart of DSPF algorithm.

The approximate conditional density in Eq. (16) is equivalently maximized by minimizing its negative logarithm subject to nonlinear constraints. The corresponding optimization problem is posed as

$$
\begin{aligned}
\bar{x}_{k}= & \left.\underset{x_{k}}{\operatorname{argmin}} x_{k}-\tilde{x}_{k}\right) \underset{\tilde{P}_{k}^{-1}}{2}+\| y_{k}-h\left(x_{k}\right)_{R^{-1}}^{2}, \\
& \text { s.t. } x_{k} \in \mathbb{R}_{\Sigma}^{n} \cap \mathbb{X} .
\end{aligned}
$$

If the a priori covariance $\tilde{P}_{k}$ is projected on constraints, then $\tilde{P}_{k}^{p}$ may be singular in which case $\tilde{x}_{k}^{p}$ and $\tilde{P}_{k}$ can be used (Simon, 2010). This approach is referred to as MAP-DSPF. It is equivalent to an implementation of MHE in a horizon of one with direct sampling particle filter used to compute the constrained arrival cost parameters.

\subsection{Direct sampling particle filter algorithm}

The algorithm for a particle filter based on direct sampling from an approximate conditional pdf including linear and nonlinear constraints is listed below with an accompanying flow chart in Fig. 1.

1. At $k$, propagate previous conditional sample set $\left\{\hat{x}_{k-1}^{i}\right\}_{i=1}^{N}$ through system model to generate a priori sample set $\left\{\tilde{x}_{k}^{i}\right\}_{i=1}^{N}$. In case of nonlinear constraints, apply projection operation on violating samples. 
2. Compute a priori sample mean $\tilde{x}_{k}$ and covariance $\tilde{P}_{k}$. Apply projection to the moments if linear or nonlinear constraints $\mathbb{X}$ are present.

3. Uniformly draw bank of candidate samples $\left\{x_{k}^{i}\right\}_{i=1}^{N}$ from support $\mathbb{R}_{\Sigma}^{n}$ using bounds by 3-sigma rule. Restrict sampling to state space $\mathbb{R}_{\Sigma}^{n} \cap \mathbb{X}$ if constraints are present.

4. Compute normalized values of approximate conditional pdf $p_{\mathbb{X}}^{*}\left(x_{k}^{i} \mid Y_{1: k}\right)$ and discrete cumulative distribution function supported over candidates $\left\{x^{i}\right\}_{i=1}^{N}$.

5. For $i=1, \ldots, N$, draw conditional samples for $\left\{\hat{x}_{k}^{i}\right\}_{i=1}^{N}$ from bank of candidate samples $\left\{x_{k}^{i}\right\}_{i=1}^{N}$ according to discrete cdf.

6. Compute conditional mean $\hat{x}_{k}$ and covariance $\hat{P}_{k}$ for state estimation. In case of linear or no constraints go to step 7 .

For nonlinear constraints either (a) apply projection operation on moments or (b) solve constrained MAP problem on approximate conditional pdf for mode estimate $\bar{x}_{k}$,

7. Set $k=k+1$. Go to step 1 .

\section{Simulation examples}

\subsection{Linear inequality constraints}

The following gas-phase irreversible reaction of species A to species B occurs in a well mixed, constant volume isothermal batch reactor: (Haseltine \& Rawlings, 2003),

$2 \mathrm{~A} \stackrel{k=0.16}{\rightarrow} \mathrm{B}$.

The dynamics of the species partial pressures $p_{\mathrm{A}}$ and $p_{\mathrm{B}}$ are governed by a pair of nonlinear ordinary differential equations (ODEs),

$\frac{\mathrm{d}}{\mathrm{d} t}\left[\begin{array}{l}p_{\mathrm{A}} \\ p_{\mathrm{B}}\end{array}\right]=\left[\begin{array}{r}-2 k p_{\mathrm{A}}^{2} \\ k p_{\mathrm{A}}^{2}\end{array}\right]$.

The ODEs are numerically integrated by Euler method starting from initial conditions $p_{\mathrm{A}, 0}=3$ and $p_{\mathrm{B}, 0}=1$. The partial pressures are non-negative quantities, hence, the state vector is subject to the following inequality constraint:

$x \geq 0, \quad \forall t$.

where $x=\left[p_{\mathrm{A}} p_{\mathrm{B}}\right]^{\mathrm{T}}$. Discrete measurements of total pressure in the reactor $\Pi_{k}$ are sampled at intervals of $\Delta t=0.1 \mathrm{~min}$. The noisy pressure measurements are simulated as a perturbed sum of the partial pressures

$\Pi_{k}=C x_{k}+v_{k}$

where $C=\left[\begin{array}{ll}1 & 1\end{array}\right]$ and $v_{k} \sim N\left(0,0.1^{2}\right)$. There is no process noise in the simulation of partial pressures, but $w_{k} \sim \mathcal{N}\left(0,10^{-6} I_{2}\right)$ is used by the filters, where $I_{2}$ is identity matrix of size two.

For comparison, the constrained ensemble Kalman filter (CEnKF) is implemented. Since the measurement and constraints are linear, the ensemble of predicted samples $\left\{\tilde{x}_{k}^{i}\right\}_{i=1}^{N}$ in Eq. (11) is updated with the measurement $y_{k}$ by solving the following quadratic program for each particle (Prakash et al., 2010):

$$
\begin{aligned}
\hat{x}_{k}^{i}= & \underset{x_{k}^{i}}{\operatorname{argmin}} \frac{1}{2} x_{k}^{i}{ }^{\mathrm{T}} H_{k} x_{k}^{i}+c_{k}^{\mathrm{T}} x_{k}^{i}, \\
& \text { s.t. }-x_{k}^{i} \leq 0, \quad i=1, \ldots, N,
\end{aligned}
$$

where

$$
\begin{aligned}
& H_{k}=C^{\mathrm{T}} R^{-1} C+\tilde{P}_{k}^{-1}, \\
& c_{k}=-\left(C^{\mathrm{T}} R^{-1}\left(\Pi_{k}-v_{k}^{i}\right)+\tilde{P}_{k}^{-1} \tilde{x}_{k}^{i}\right),
\end{aligned}
$$
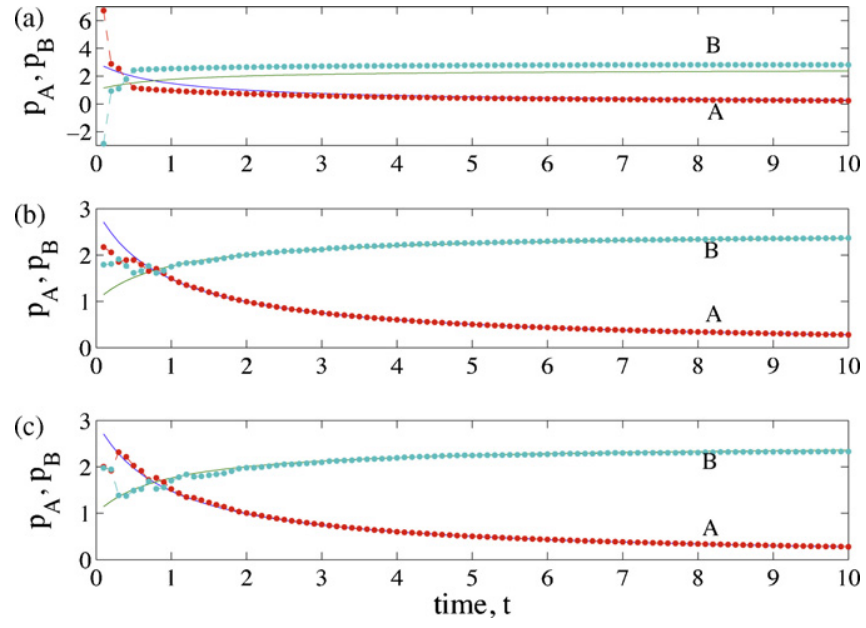

Fig. 2. Solid lines are true partial pressures, (a) particle filter estimates, (b) direct sampling particle filter estimates and (c) constrained ensemble Kalman filter estimates.

and $v_{k}^{i}$ are the samples drawn from the measurement noise pdf. Sample statistics of the updated ensemble provide the state estimates.

Moving horizon estimation is implemented in a horizon of $m=4$ using the following nonlinear optimization problem:

$$
\begin{aligned}
\left\{\bar{x}_{j}\right\}_{j=k-3}^{k}= & \underset{\left\{x_{j}\right\}_{j=k-3}^{k}}{\arg \min _{k-3}-\tilde{x}_{k-3}} \underset{\tilde{P}_{k-3}^{-1}}{2}+{ }_{j=k-3}^{k-1} x_{j+1}-f\left(x_{j}\right)_{Q^{-1}}^{2} \\
& +\quad y_{j=k-3}^{k} y_{j}-C x_{j}{ }_{R}^{2-1}, \\
& \text { s.t. }\left\{x_{j}\right\}_{j=k-3}^{k} \geq 0,
\end{aligned}
$$

where the state transition function $f$ is implemented by numerical integration of the system ODEs. The arrival cost parameters $\tilde{x}_{k-3}$ and $\tilde{P}_{k-3}$ are computed using the traditional EKF filtering update as well as the proposed DSPF update for comparison.

State estimation by all the filters is initialized by poorly known information about the initial condition, $\hat{p}_{\mathrm{A}, 0}=0.1, \hat{p}_{\mathrm{B}, 0}=4.5$ and $\hat{P}_{0}=6^{2} I_{2}$ indicating low confidence on the information. The number of samples in all the particle filters is $N=150$. The simulations are performed in Matlab on 3.2 GHz Dual-Core Intel Xeon processor running 64-bit Linux. The constrained optimization in C-EnKF and MHE is performed by the functions quadprog and fmincon respectively.

The performance of the state estimation methods is compared using the sum of squared estimation errors (SSEE), defined as

$$
\text { SSEE }=x_{k=1}^{K}\left(x_{k}(i)-\hat{x}_{k}(i)\right)^{2}, \quad i=1, \ldots, n,
$$

where $K$ is the number of measurements and $n$ is the length of the state vector.

Results of the estimation of species partial pressures from total pressure measurements by the particle filter are shown in Fig. 2(a). The estimates are slow to converge to the true dynamics and negative values for the partial pressure of species $B$ are meaningless. Such estimates by EKF are also known to converge to wrong steady states when the non-negativity constraints are ignored (Haseltine \& Rawlings, 2003). Similarly poor performance by the unconstrained EnKF is noted even when sample size is increased (Prakash et al., 2010). Fig. 2(b) and (c) displays the estimates obtained from the direct sampling particle 
Table 1

Estimation of partial pressures. Average error and computation time over 100 realizations.

\begin{tabular}{lccc}
\hline Estimation method & SSEE & & $\begin{array}{l}\text { Computation time per } \\
\text { measurement (ms) }\end{array}$ \\
\cline { 2 - 3 } & \multicolumn{2}{c}{$p_{\mathrm{A}}$} & $p_{\mathrm{B}}$ \\
\hline PF & 34 & 73 & 0.4 \\
DSPF & 1.44 & 2.20 & 0.5 \\
C-EnKF (quadprog) & 1.76 & 2.14 & 170 \\
C-EnKF (block quadprog) & 1.76 & 2.10 & 118 \\
MHE w/ EKF ( $m=4$, fmincon) & 1.92 & 2.70 & 12 \\
MHE w/ DSPF (m=4, fmincon) & 0.85 & 1.10 & 11 \\
\hline
\end{tabular}

filter and C-EnKF, respectively, where constraints are explicitly enforced.

In Table 1 , average values over 100 realizations for SSEE and computation time per measurement are shown. The estimation error performance of DSPF is similar to C-EnKF. The computation time for DSPF remained about the same as the PF, whereas C-EnKF is more time consuming due to the $N=150$ quadratic programs, each solving for the $n=2$ dimensional state vector. The error and computational performance of C-EnKF is in line with that reported recently for this simulation example (Prakash et al., 2010).

In an alternate implementation of C-EnKF, a single quadratic program with $N n$ decision variables is solved to update the ensemble at each time instance. The a priori ensemble of $N$ samples is appended into a single vector of size $N n \times 1$. Similarly, the perturbed measurements $y_{k}-v_{k}^{i}, i=1, \ldots, N$ are appended into an $N p \times 1$ vector. The appropriate measurement matrix, measurement noise covariance matrix and a priori state covariance matrix are block diagonal matrices with $N$ repeated elements in each. The computational time shown in Table 1 for the block quadratic programming implementation of C-EnKF is about 30\% smaller.

The MHE using EKF update for arrival cost also performed similar to C-EnKF, but with a much smaller computational time due to a single nonlinear optimization involving $m n=8$ decision variables at each time instance. The MHE using DSPF for arrival cost noticeably reduced the estimation error at about the same computational time, suggesting improved computation of arrival cost and quick recovery from poor initialization in the horizon. Typical sample paths in Fig. 3 show that MHE with DSPF estimates converged faster than MHE with EKF estimates.
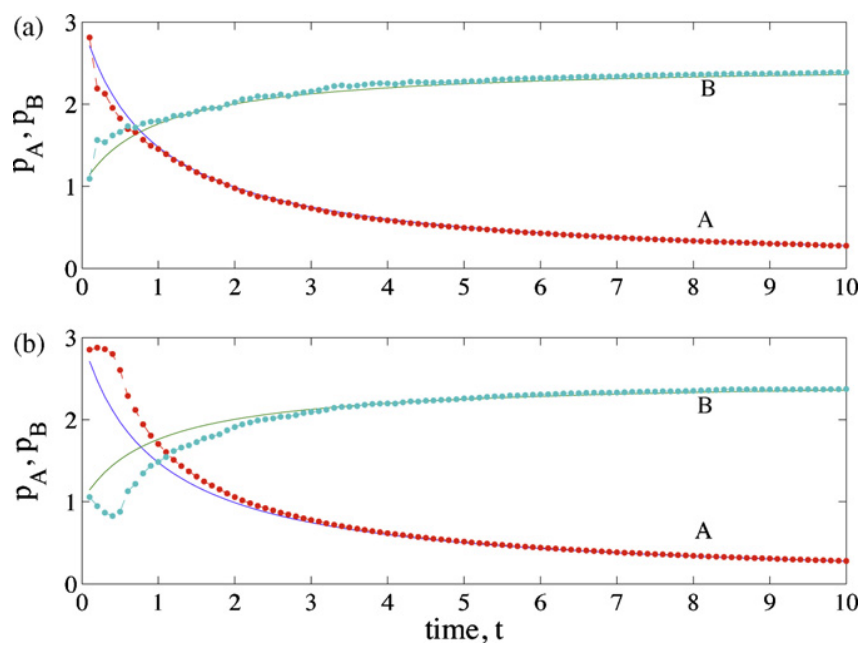

Fig. 3. Solid lines are true partial pressures, (a) MHE $(m=4)$ estimates with DSPF for arrival cost and (b) MHE $(m=4)$ estimates with EKF for arrival cost.
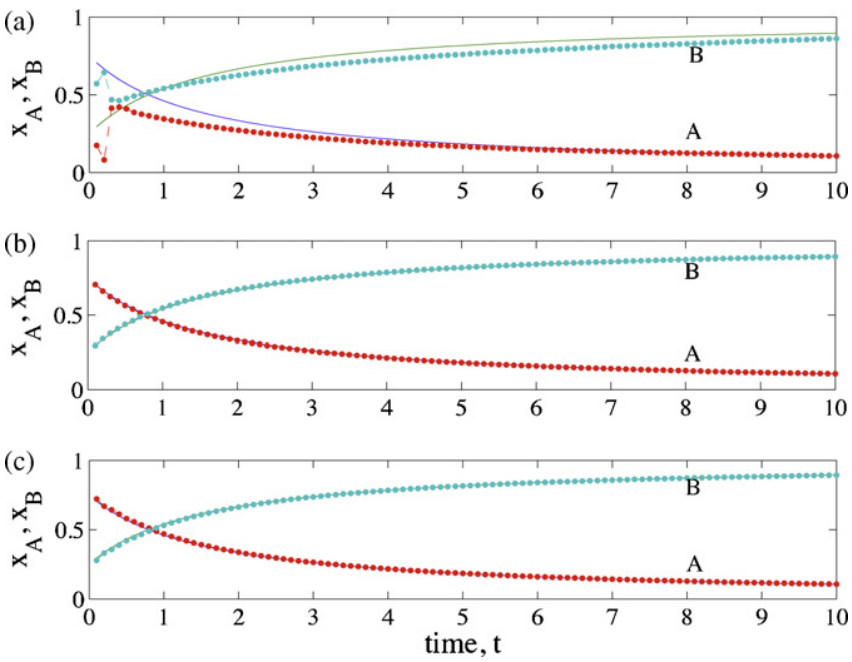

Fig. 4. Solid lines are true mole fractions, (a) particle filter estimates, (b) direct sampling particle filter estimates and (c) constrained ensemble Kalman filter estimates.

\subsection{Linear equality constraints}

The dynamics of the gas-phase species mole fractions in the batch reactor, $x_{\mathrm{A}}$ and $x_{\mathrm{B}}$, are described by the following pair of ODEs:

$\frac{\mathrm{d}}{\mathrm{d} t} \quad \begin{gathered}x_{\mathrm{A}} \\ x_{\mathrm{B}}\end{gathered}=\begin{array}{r}-5 k x_{\mathrm{A}}^{2} \\ 5 k x_{\mathrm{A}}^{2}\end{array}$

with the particular initial conditions $x_{\mathrm{A}, 0}=0.75$ and $x_{\mathrm{B}, 0}=0.25$. The mole fractions by nature obey the following linear constraints:

$A x=1, \quad x \geq 0, \quad \forall t$,

where $x=\left[x_{\mathrm{A}} x_{\mathrm{B}}\right]^{\mathrm{T}}$ and $A=\left[\begin{array}{ll}1 & 1\end{array}\right]$. The total reactor pressure ${ }_{k}$, sampled at $\Delta t=0.1 \mathrm{~min}$ intervals, is a nonlinear function of the mole fractions,

$$
k=\frac{5}{x_{\mathrm{A}, k}+2 x_{\mathrm{B}, k}}+v_{k},
$$

with $v_{k} \sim N\left(0,0.1^{2}\right)$. The mole fractions are simulated as noise free but the filters use $w_{k} \sim \mathcal{N}\left(0,10^{-6} I_{2}\right)$.

The C-EnKF algorithm is implemented by solving the following nonlinear optimization for each particle:

$$
\begin{gathered}
\left.\hat{x}_{k}^{i}=\underset{x_{k}^{i}}{\operatorname{argmin}} x_{k}^{i}-\tilde{x}_{k}^{i}\right) \underset{\tilde{P}_{k}^{-1}}{2}+\| \quad k-h\left(x_{k}^{i}\right)-v_{k}^{i}{ }_{R^{-1}}^{2}, \\
\text { s.t. } A x_{k}^{i}=1, \quad x_{k}^{i} \geq 0, \quad i=1, \ldots, N,
\end{gathered}
$$

where $h$ is the nonlinear measurement function in Eq. (36). Using $N=150$ samples, the filters are initialized by $\hat{x}_{0}=x_{0}$ and $\hat{P}_{0}=I_{2}$.

From the PF results shown in Fig. 4(a) it is seen that the estimates are slow to converge and violated the sum to unity constraint. Estimates from DSPF and C-EnKF shown in Fig. 4(b) and (c), respectively, converged almost immediately to true dynamics and they are also verified to obey the constraints. In Table 2, the average values of SSEE and computation time show that the performance

Table 2

Estimation of mole fractions. Average error and computation time over 100 realizations.

\begin{tabular}{lllc}
\hline Estimation method & SSEE & & $\begin{array}{l}\text { Computation time per } \\
\text { measurement (ms) }\end{array}$ \\
\cline { 2 - 3 } & $x_{\mathrm{A}}$ & $x_{\mathrm{B}}$ & \\
\hline PF & 0.74 & 0.38 & 0.4 \\
DSPF & 0.0035 & 0.0035 & 0.5 \\
C-EnKF (fmincon) & 0.0028 & 0.0028 & 1100 \\
\hline
\end{tabular}




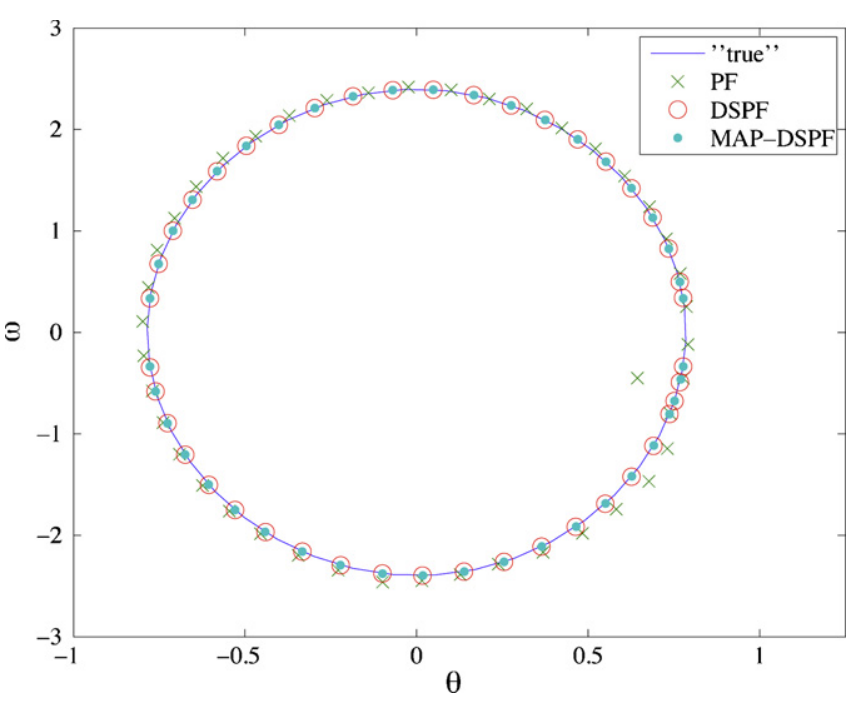

Fig. 5. The oval in solid line is the constrained state space trajectory of the pendulum for approximately one period. Plotted are mean estimates of angular position and velocity by particle filter $(x)$, direct sampling particle filter $(0)$ and mode estimates from MAP-DSPF $(\bullet)$.

improvement of DSPF over PF is obtained at about the same computational cost of the PF. However, due to the $N=150$ nonlinear optimizations performed for the ensemble update in C-EnKF, it required large computational time.

\subsection{Nonlinear equality constraints}

The dynamics of a frictionless, unforced simple pendulum are governed by the following set of ODEs (Simon, 2010):

$\frac{\mathrm{d}}{\mathrm{d} t} \quad \begin{gathered}\omega \\ =\end{gathered} \frac{g^{\omega}}{l} \sin \theta$,

where $\theta$ is the angular position, $\omega$ is the angular velocity, $g$ is the acceleration due to gravity and $l$ is the length of the pendulum. The differential equations are numerically integrated using ode 45 function, from initial conditions $\theta_{0}=\pi / 4$ and $\omega_{0}=0$. The state variables $\theta$ and $\omega$ are constrained by conservation of energy according to

$-m g l \cos \theta+\frac{1}{2} m l^{2} \omega^{2}=E_{0}, \quad \forall t$,

where $m$ is the mass of the bob and $E_{0}$ is the total energy, which is a constant determined by the initial angular position and pendulum parameters. For this simulation, let $l=1, g=9.81, m=1$ and $E_{0}=-g \cos \theta_{0}$.

The angular position and velocity are sampled at $\Delta t=0.05$ time intervals for approximately the duration of one period of the pendulum's oscillations according to

$y_{k}=\begin{gathered}\theta_{k} \\ \omega_{k}\end{gathered}+v_{k}$

with $v_{k} \sim N\left(0,0.1^{2} I_{2}\right)$. The system dynamics are noise free but the filters use $w_{k} \sim \mathcal{N}\left(0,0.007^{2} I_{2}\right)$. State estimation is initiated with $\hat{x}_{0}=$ $x_{0}$ and $\hat{P}_{0}=I_{2}$. One hundred samples are used in the particle filters.

The estimated values of the angular position and velocity are shown in Fig. 5 in the state space, where the oval in solid line is the restricted path of the pendulum simulated for approximately one period. The PF estimates plotted in $(x)$ are unaware of the nonlinear constraint, therefore deviated from the constrained path. The unprojected mean estimate provided by DSPF plotted in ( 0 ) is considerably closer to the path, but the mean does not respect the nonlinear constraint. This is evident from Fig. 6, where panel (a) (a)
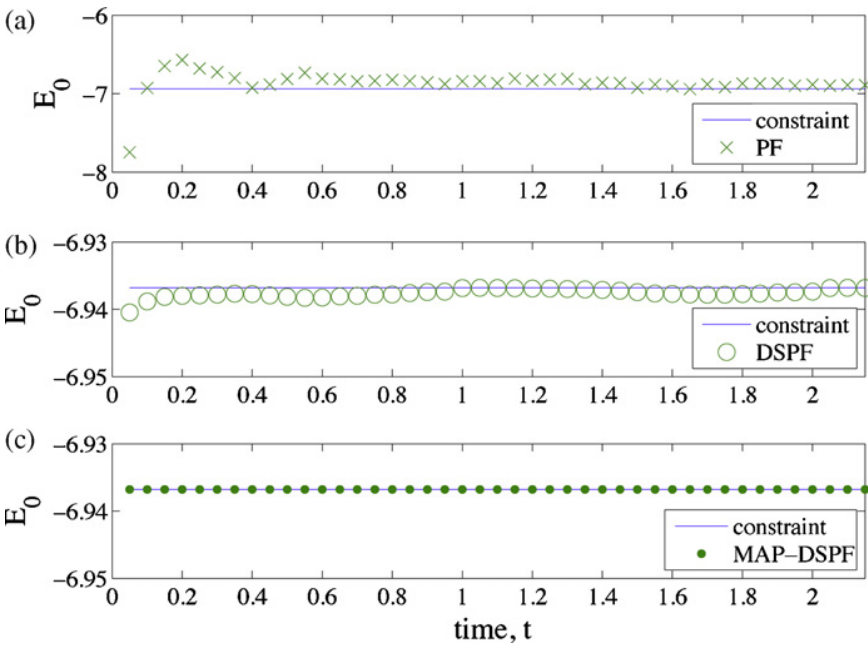

Fig. 6. The value of constant total energy $E_{0}$ computed from (a) PF mean estimates, (b) DSPF mean estimates and (c) MAP-DSPF mode estimates.

shows the violation of constraints by PF estimates and panel (b) shows the constraint value of DSPF mean estimates, which is close but not equal to the constant total energy $E_{0}$. Finally, in Fig. 5 the MAP-DSPF estimates of the constrained mode is plotted in $(\bullet)$, all of which lie on the restricted path. This fact is also verified in Fig. 6(c) that the constraint is satisfied at all times.

Average SSEE and computation time are listed in Table 3. The computation time for PF and DSPF are again similar. In the constrained mode estimation by MAP-DSPF, the quadratic objective function is subject to nonlinear constraints, which is handled by the fmincon function in Matlab. The time shown for MAP-DSPF includes the time to solve the optimization problem as well as the time used to update the samples.

\subsection{Poor performance of DSPF}

The following univariate time-varying nonlinear system is widely used as a bench mark problem in particle filter literature (Gordon et al., 1993):

$x_{k}=\frac{x_{k-1}}{2}+\frac{25 x_{k-1}}{1+x_{k-1}^{2}}+8 \cos (1.2(k-1))+w_{k-1}$,

$y_{k}=\frac{x_{k}^{2}}{20}+v_{k}$

with $w_{k-1} \sim \mathcal{N}(0,10), v_{k} \sim \mathcal{N}(0,1)$ and initial condition $x_{0}=1$. Estimation is initiated with a priori information $\hat{x}_{0}=1, \hat{P}_{0}=1$ and $N=100$ particles.

The likelihood function for this system is a symmetrical bimodal function peaking at $\pm \sqrt{20 y_{k}}$ for positive measurements, which is the case most of the time. When the measurement is negative, the likelihood function is unimodal and centered on zero. The true a priori density is typically an asymmetric bimodal function that accentuates one of the modes of the likelihood while suppressing

Table 3

Estimation of angular position and angular velocity. Average error and computation time over 100 realizations.

\begin{tabular}{llll}
\hline Estimation method & SSEE & & $\begin{array}{l}\text { Computation time per } \\
\text { measurement (ms) }\end{array}$ \\
\cline { 2 - 3 } & $\theta$ & $\omega$ & \\
\hline PF & 0.04 & 0.09 & 5 \\
DSPF & 0.0053 & 0.062 & 7 \\
MAP-DSPF ( $m=1$, fmincon $)$ & 0.0050 & 0.057 & 35 \\
\hline
\end{tabular}


Table 4

Estimation of unconstrained system. Average error and computation time over 100 realizations.

\begin{tabular}{lcc}
\hline Estimation method & SSEE & $\begin{array}{l}\text { Computation time per } \\
\text { measurement (ms) }\end{array}$ \\
\hline PF & 1124 & 0.4 \\
DSPF & 2286 & 0.5 \\
C-EnKF (fminunc) & 3623 & 460 \\
\hline
\end{tabular}
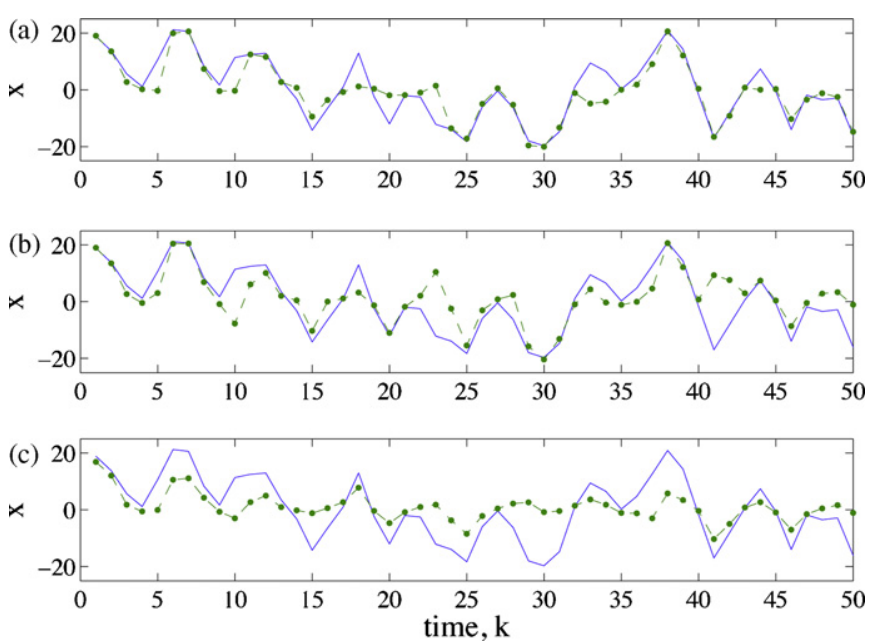

Fig. 7. Solid line is true state (unconstrained system), (a) particle filter estimates, (b) direct sampling particle filter estimates and (c) constrained ensemble Kalman filter estimates.

the other for the conditional density. The Gaussian approximation leads to poor performance in DSPF for this system. The average SSEE shown in Table 4 for DSPF are significantly larger than that of PF. The C-EnKF implemented by using fminunc also performed poorly in this case. Representative estimates by the three filters are shown in Fig. 7.

\section{Conclusions}

The Gaussian probability density function (pdf) serves as a popular, convenient approximation for the a priori pdf. The arrival cost term in moving horizon estimation (MHE) is typically determined by a Gaussian. The general particle filtering approach explicitly discards any assumptions on the a priori density. In this paper it is shown that by including the Gaussian assumption it is possible to draw samples directly from an approximate conditional density. The sampling is restricted to linear constraints to obtain constrained conditional mean and covariance. The direct sampling algorithm uses the MAP formulation for estimating the constrained mode under nonlinear constraints. Alternatively, projection techniques are used to enforce nonlinear constraints on the mean estimate. Simulation results indicate that the proposed method performed as well as optimization based approaches with samples, while retaining the small computational load of the particle filter.

\section{Acknowledgement}

This material is based upon work supported by the National Science Foundation under Grant No. CTS-0522864.

\section{Appendix A.}

Given the mean $\tilde{x}$ that violates the constraints $A x=b$ or $A x \geq b$ its projection $\tilde{x}^{p}$ on the constraints boundary is sought in a least squares or minimum Euclidean norm sense as

$$
\begin{aligned}
\tilde{x}^{p}=\mathbf{p}(\tilde{x})= & \underset{x}{\operatorname{argmin}}(\tilde{x}-x)^{\mathrm{T}}(\tilde{x}-x), \\
& \text { s.t. } A x=b .
\end{aligned}
$$

The constraints are incorporated by considering the Lagrangian form

$J=(\tilde{x}-x)^{\mathrm{T}}(\tilde{x}-x)+2 \lambda^{\mathrm{T}}(b-A x)$.

Solving the optimality conditions $\partial J / \partial x=\partial J / \partial \lambda=0$, yields the following

$\tilde{x}^{p}=\tilde{x}+A^{\mathrm{T}} \lambda$,

$\lambda=\left(A A^{\mathrm{T}}\right)^{-1}(b-A \tilde{x})$.

Therefore, the projected mean $\tilde{x}^{p}$ and the corresponding covariance $\tilde{P}^{p}$ are

$\tilde{x}^{p}=\tilde{x}+A^{\mathrm{T}}\left(A A^{\mathrm{T}}\right)^{-1}(b-A \tilde{x})$,

$\tilde{P}^{p}=\left(I-A^{\mathrm{T}}\left(A A^{\mathrm{T}}\right)^{-1} A\right) \tilde{P}\left(I-A^{\mathrm{T}}\left(A A^{\mathrm{T}}\right)^{-1} A\right)^{\mathrm{T}}$.

The projection operator $\mathbf{p}$ is a translated linear transformation.

More generally, when considering the covariance matrix $\tilde{P}$ also, a maximum likelihood projection operation is posed as

$$
\begin{aligned}
\tilde{x}^{p}= & \mathbf{p}(\tilde{x})=\underset{x}{\operatorname{argmin}}(\tilde{x}-x)^{\mathrm{T}} \tilde{P}^{-1}(\tilde{x}-x), \\
& \text { s.t. } A x=b,
\end{aligned}
$$

that results in

$$
\begin{aligned}
& \tilde{x}^{p}=\tilde{x}+\tilde{P}^{-1} A^{\mathrm{T}}\left(A \tilde{P}^{-1} A^{\mathrm{T}}\right)^{-1}(b-A \tilde{x}), \\
& \tilde{P}^{p}=\left(I-\tilde{P}^{-1} A^{\mathrm{T}}\left(A \tilde{P}^{-1} A^{\mathrm{T}}\right)^{-1} A\right) \tilde{P}\left(I-\tilde{P}^{-1} A^{\mathrm{T}}\left(A \tilde{P}^{-1} A^{\mathrm{T}}\right)^{-1} A\right)^{\mathrm{T}} .
\end{aligned}
$$

\section{References}

Arulampalam, M. S., Maskell, S., Gordon, N., \& Clapp, T. (2002). A tutorial on particle filters for online nonlinear/non-Gaussian Bayesian tracking. IEEE Transactions on Signal Processing, 50(2), 174-188.

Botchu, S. (2006). Nonlinear model predictive control with particle filter for state estimation. M.S. thesis, Cleveland State University, Cleveland, $\mathrm{OH}$

Chen, W. S., Bakshi, B. R., Goel, P. K., \& Ungarala, S. (2004). Bayesian estimation via sequential Monte Carlo sampling: unconstrained nonlinear dynamic systems. Industrial \& Engineering Chemistry Research, 43, 4012-4025.

Gordon, N., Salmond, D. J., \& Smith, A. F. M. (1993). Novel approach to nonlinear/nonGaussian Bayesian state estimation. IEE Processinds-F, 140(2), 107-113.

Haseltine, E. L., \& Rawlings, J. B. (2003). A critical evaluation of extended Kalman filtering and moving horizon estimation. Technical report, TX-WI Modeling and Control Consortium. http://jbrwww.che.wisc.edu/tech-reports/twmcc-2002-03.pdf.

Julier, S., \& LaViola, J. (2007). On Kalman filtering with nonlinear equality constraints. IEEE Transactions on Signal Processing, 55, 2774-2784.

Kandepu, R., Foss, B., \& Imsland, L. (2008). Applying the unscented Kalman filter for nonlinear state estimation. Journal of Process Control, 18, 753-768.

Kolas, S., Foss, B. A., \& Schei, T. S. (2009). Constrained nonlinear state estimation based on the UKF approach. Computers E Chemical Engineering 33, 1386-1401.

Lang, L., Chen, W. S., Bakshi, B. R., Goel, P. K., \& Ungarala, S. (2007). Bayesian estimation via sequential Monte Carlo sampling-Constrained dynamic systems. Automatica, 43(9), 1615-1622.

Lang, L., Goel, P. K., \& Bakshi, B. R. (2006). A smoothing based method to improve performance of sequential Monte Carlo estimation under poor prior. In Chemical process control, CPC7 Lake Louise, Alberta, Canada,

Lang, L., Zhang, X., Goel, P. K. \& Bakshi, B. R. (2005). Estimation in nonlinear dynamic systems via Monte Carlo sampling versus moving horizon estimation-Complementary or competitive? In Annual meeting of AIChE Cincinnati, $\mathrm{OH}$.

Narasimhan, S., \& Rengaswamy, R. (2009). Reply to comments on "robust and reliable estimation via unscented recursive nonlinear dynamic data reconciliation" (URNDDR). Journal of Process Control, 19, 719-721.

Prakash, J., Patwardhan, S. C., \& Shah, S. L. (2008). Constrained state estimation using particle filters. In Proceedings of the 17th IFAC world congress Seoul, Korea, (pp. 6472-6477)

Prakash, J., Patwardhan, S. C., \& Shah, S. L. (2010). Constrained nonlinear state estimation using ensemble Kalman filters. Industrial \& Engineering Chemistry Research, $49,2242-2253$.

Qu, C., \& Hahn, J. (2009). Computation of arrival cost for moving horizon estimation via unscented Kalman filtering. Journal of Process Control, 19, 358-363.

Rajamani, M. R., \& Rawlings, J. B. (2007). Improved state estimation using a combination of moving horizon estimator and particle filters. In Proceedings of the American control conference New York, NY, (pp. 4443-4444). 
Rao, C. V., \& Rawlings, J. B. (2002). Constrained process monitoring: Moving-horizon approach. AIChE Journal, 48(1), 97-109.

Rawlings, J. B., \& Bakshi, B. R. (2006). Particle filtering and moving horizon estimation. Computers \& Chemical Engineering, 30, 1529-1541.

Robertson, D. G., \& Lee, J. H. (1995). A least squares formulation for state estimation Journal of Process Control, 5(4), 291-299.

Robertson, D. G., Lee, J. H., \& Rawlings, J. B. (1996). A moving horizon-based approach to least squares estimation. AIChE Journal, 42(8), 2209.

Shao, X., Huang, B., \& Lee, J. M. (2010). Constrained Bayesian state estimation-A comparative study and a new particle filter based approach. Journal of Process Control, 20, 143-157.

Simon, D. (2010). Kalman filtering with state constraints: A survey of linear and nonlinear algorithms. IET Control Theory Applications, 4(8), 1303-1318.
Teixeira, B. O., Torres, L. A. B., Aguirre, L. A., \& Bernstein, D. S. (2010). On unscented Kalman filtering with state interval constraints. Journal of Process Control, 20 45-57.

Ungarala, S. (2009). Computing arrival cost parameters in moving horizon estimation using sampling based methods. Journal of Process Control, 19, 15761588.

Ungarala, S., Li, K., \& Chen, Z. (2008). Constrained Bayesian state estimation using a cell filter. Industrial E' Engineering Chemistry Research, 47(19), 7312 7322

Vachhani, P., Narasimhan, S., \& Rengaswamy, R. (2006). Robust and reliable estimation via unscented recursive nonlinear dynamic data reconciliation. Journal of Process Control, 16, 1075-1086. 